\title{
Type II toxin: antitoxin systems. More than small selfish entities?
}

\author{
Andrea Rocker $^{1} \cdot$ Anton Meinhart $^{1}$
}

Received: 7 November 2015 / Accepted: 9 November 2015 / Published online: 23 November 2015

(C) The Author(s) 2015. This article is published with open access at Springerlink.com

\begin{abstract}
Toxin-antitoxin (TA) modules regulate metabolism and viability of bacteria and archaea. In type II TA systems these functions are generally thought to be performed by two small proteins. However, evidence is increasing that the toxins are much more diverse and can form multidomain proteins. Recently, we published a novel type II TA system in which toxin and antitoxin are covalently linked into a single polypeptide chain. In this review we summarize the current knowledge on these elongated toxin homologs and provide perspectives for future study.
\end{abstract}

Keywords Toxin-antitoxin system · Fusion ·

Multi-domain protein $\cdot$ Elongated toxin

Bacterial type II toxin-antitoxin (TA) systems were already identified in 1983 as bicistronic operons that ensure stable plasmid maintenance in bacteria (Ogura and Hiraga 1983). They are generally thought to consist of a small harmful enzyme (toxin) and a small proteinaceous antidote (antitoxin) (Goeders and Van Melderen 2014; Yamaguchi et al. 2011). Upon binding to each other, a neutral protein-protein TA-complex is formed under non-stress condition that remains inactive in the cytoplasm of the producing bacterial organism. Cellular concentrations of type II TA complexes are often regulated by autoregulation of transcription by the antitoxin or toxin-antitoxin complex (Gerdes

Communicated by M. Kupiec.

Anton Meinhart

Anton.Meinhart@mpimf-heidelberg.mpg.de

1 Department of Biomolecular Mechanisms, Max

Planck Institute for Medical Research, Jahnstrasse 29,

69120 Heidelberg, Germany and Maisonneuve 2012) and simple repression by promoter binding is in some cases further enhanced by conditional cooperativity (Garcia-Pino et al. 2010). Activation of the toxin is accomplished by degradation of the antitoxin by cellular proteases liberating the proteolytically stable toxin (Schuster and Bertram 2013). By this simple mechanism, plasmid encoded type II TA systems lead to post-segregational cell death in bacterial progeny that are devoid of the plasmid. Although originally thought to mainly serve by this mechanism as addiction modules that ensure the maintenance of bacterial populations containing stably inherited plasmids (Engelberg-Kulka and Glaser 1999), type II TA systems are also now considered as important modulators of bacterial physiology for instance as stress response loci (Gerdes et al. 2005, Wang and Wood 2011). Moreover, type II TA systems are highly prevalent on the chromosome of bacteria and additional functions apart from being simple selfish entities that secure stable maintenance of mobile genetic elements are reported (Van Melderen and Saavedra De Bast 2009). In fact, four additional different roles have been recently been attributed to type II TA systems which are still a matter of debate in some cases (Van Melderen 2010); these functions range from being modules that induce programmed-cell death activated by a variety of unrelated stressful conditions where altruistic death provide nutriments for the remaining bacteria as reported for the chromosomal encoded mazEF locus from Escherichia coli (Engelberg-Kulka et al. 2006), modules that lead to global inhibition of translation as it is the case for the relBE locus from E. coli (Christensen et al. 2001), modules such as the hipAB from $E$. coli locus that induces persistence of a small fraction of bacteria (Correia et al. 2006, Korch et al. 2003) upon phosphorylation of the glutamyltRNA synthetase (Germain et al. 2013; Kaspy et al. 2013) to developmental regulators as reported for the $m a z E F$ 
locus from Myxococcus xanthus (Nariya and Inouye 2008). Additionally, other type II TA systems have been shown to influence biofilm formation (Kim et al. 2009) or virulence (Harvey et al. 2011). Noteworthy, in addition to the here exemplified type II TA systems, similar functions have been ascribed for a number of other type II TA systems as well, and even overlapping functions have been proposed for some systems (De la Cruz et al. 2013; Magnuson 2007; Van Melderen and Saavedra De Bast 2009; Wang and Wood 2011). Similar as type II TA systems are diverse in their function their cognate toxins also interfere with a number of important cellular processes. Type II TA systems interfere for instance with replication and transcription by inhibiting Topoisomerases (Bernard and Couturier 1992; Harms et al. 2015; Jiang et al. 2002; Maki et al. 1992) or impair replication through their interaction with the beta Sliding clamp of DNA polymerase III (Aakre et al. 2013). Interestingly, a huge number of type II TA-systems interfere with translation by either acting as RNA interferases or directly inhibiting the ribosome (Castro-Roa et al. 2013; Guglielmini and Van Melderen 2011). More recently, type II TA-systems were also shown to impair with cell wall synthesis (Mutschler et al. 2011) or cell division (Masuda et al. 2012), thereby evoking cell death or stasis. Bioinformatic analyses browsing the increasing number of available microbial genomes revealed that type II TA systems are widespread in both bacteria and archaea where they can exist in multiple copies on plasmids as well as on chromosomes (Leplae et al. 2011; Makarova et al. 2009; Sberro et al. 2013; Sevin and Barloy-Hubler 2007; Shao et al. 2011). Hitherto, type II TA systems have been grouped into 12 different families, depending on the toxin primary sequence and activity (Leplae et al. 2011). Nevertheless, the current picture on type II TA system seems to be far from complete.

The commonly used bioinformatic approach for de novo identification of type II TA system is based on the occurrence of bicistronic operons, of which the second translational product encodes a putative toxic enzyme (Leplae et al. 2011; Sberro et al. 2013; Shao et al. 2011). Homology searches have greatly advanced our knowledge of those systems and even distantly related type II TA systems have been identified. However, a more complex search for type II TA systems revealed a number of unusually long and sometimes even solitary toxin genes in the genome of different bacteria (Makarova et al. 2009; Sevin and BarloyHubler 2007). The cellular function of these novel and remarkable type II TA loci is poorly characterized and most of these systems are scientifically orphaned, so far.

One of the first examples of such long type II toxins is encoded by the phoH2 locus found in the genomes of Mycobacterium tuberculosis and Mycobacterium smegmatis, but also in the thermophilic organism Thermobispora bispora (Andrews and Arcus 2015). In contrast to all previously reported type II toxins that are small, single-domain proteins, the toxin $\mathrm{PhoH} 2$ was shown to be a two-domain protein consisting of an N-terminal VapC toxin-like PIN domain linked to a $\mathrm{C}$-terminal $\mathrm{PhoH}$ helicase domain adding up to a total molecular weight of approximately $50 \mathrm{kDa}$. The latter ATPase domain was shown to have RNA binding and unwinding activity, complementing the RNase activity of the N-terminal VapC toxin domain. $\mathrm{PhoH} 2$ is co-transcribed from a bicistronic operon together with the open reading frame of the PhoAT antitoxin that binds to $\mathrm{PhoH} 2$. Finally, expression of M. tuberculosis $\mathrm{PhoH} 2$ in M. smegmatis was reported to have a negative effect on growth, which was alleviated by the presence of the adjacently encoded PhoAT antitoxin. The authors speculate that the acquisition of additional domains expands the versatility of the mycobacterial VapBC TA systems, which function as posttranscriptional regulators in response to environmental stress (Andrews and Arcus 2015).

Another variety of unconventional and previously unrecognized type II TA system is the multi-domain and auto-regulated EzeT system encoded by a variety of different strains from Escherichia coli and several related organisms (Rocker and Meinhart 2015). We have shown that the C-terminal domain of this type II TA system harbors a zeta-toxin like UDP- $N$-acetylglucosamine kinase domain that interferes with peptidoglycan synthesis similar to the related toxins from Streptococcous pneumoniae and Streptococcus pyogenes (Mutschler et al. 2011; Tabone et al. 2014). In contrast to conventional type II TA systems, EzeT contains an N-terminal antitoxin domain that is fused with the kinase domain (Rocker and Meinhart 2015). This covalent linkage of toxin and antitoxin functionalities within a single polypeptide chain leads to an auto-regulated toxin which had most likely evolved by fusion of a conventional bicistronic operon. The action of an antitoxin in cis abolishes passive complex diffusion and therefore provides additional possibilities for regulation. Activation could be either achieved by protease cleavage, partial digestion, chaperone binding or protein modification. Moreover, the generally observed recovery of a toxinpoisoned bacterial population by newly synthesized antitoxin until the "point of no return" is not feasible in such systems, as toxin and antitoxin are synthesized in equimolar amounts and are additionally fused. Interestingly, a similar fusion protein TA system has been reported for the Phd antitoxin and a RelE toxin, yb_293189 TA system from Ralstonia eutropha (Loris and Garcia-Pino 2014). However, in this case truncations are thought to abolish enzymatic activity of the toxin as well as the inhibitory function of the antitoxin. Whether this system is a deadend product in evolution or constitutes a functional system awaits further investigations. 
Furthermore, additional evidence for an expansion of toxin functions is provided by domain analyses in the protein family database PFAM (Finn et al. 2014). For example, multi-domain zeta toxin homologs (PF06414) are predicted but so far uncharacterized. In this family members are annotated in which the zeta core regions of roughly 200 amino acids are predicted to be linked to phosphatase or peptidoglycan binding domains. In addition, zeta homologs with more than 500 amino acids have been reported previously (Chan et al. 2012). However, no experimental studies of these systems have been performed, yet. Similarly, ParE-like toxins (PF05016), Fic/Doc-domains (PF02661) and PemK family toxins (PF02452) have been described as parts of multi-domain proteins. In addition, 200 amino acid long proteins combining a 60 amino acid long N-terminal region with homology to the toxin HicA (PF07927) with a 130 amino acid long region homologous to the HicB-like antitoxin (PF15919) as well as an HicB domain (PF05534) are assigned, indicating a putative TA fusion protein.

It seems that investigations of elongated toxin proteins still hold unanticipated number of surprising findings as many novel functionalities and regulatory concepts can be acquired by domain fusion, adding to the complexity of TA systems.

Acknowledgments Open access funding provided by Max Planck Society (or associated institution if applicable). We apologize to all colleagues whose excellent contributions to the field could not be mentioned due to space limitation. We thank Manuel Espinosa for critical reading of the manuscript and helpful discussions. We are grateful to I. Schlichting for continuous encouragement and support. A.M. is a member of CellNetworks, Heidelberg, and supported by the Chica and Heinz Schaller Foundation, Heidelberg.

Open Access This article is distributed under the terms of the Creative Commons Attribution 4.0 International License (http://creativecommons.org/licenses/by/4.0/), which permits unrestricted use, distribution, and reproduction in any medium, provided you give appropriate credit to the original author(s) and the source, provide a link to the Creative Commons license, and indicate if changes were made.

\section{References}

Aakre CD, Phung TN, Huang D, Laub MT (2013) A bacterial toxin inhibits DNA replication elongation through a direct interaction with the beta sliding clamp. Mol Cell 52:617-628

Andrews ES, Arcus VL (2015) The mycobacterial PhoH2 proteins are type II toxin antitoxins coupled to RNA helicase domains. Tuberculosis (Edinb) 95:385-394

Bernard P, Couturier M (1992) Cell killing by the F plasmid CcdB protein involves poisoning of DNA-topoisomerase II complexes. J Mol Biol 226:735-745

Castro-Roa D, Garcia-Pino A, De Gieter S, van Nuland NA, Loris R, Zenkin N (2013) The Fic protein Doc uses an inverted substrate to phosphorylate and inactivate EF-Tu. Nat Chem Biol 9:811-817
Chan WT, Moreno-Cordoba I, Yeo CC, Espinosa M (2012) Toxinantitoxin genes of the Gram-positive pathogen Streptococcus pneumoniae: so few and yet so many. Microbiol Mol Biol Rev 76:773-791

Christensen SK, Mikkelsen M, Pedersen K, Gerdes K (2001) RelE, a global inhibitor of translation, is activated during nutritional stress. Proc Natl Acad Sci USA 98:14328-14333

Correia FF, D’Onofrio A, Rejtar T, Li L, Karger BL, Makarova K, Koonin EV, Lewis K (2006) Kinase activity of overexpressed HipA is required for growth arrest and multidrug tolerance in Escherichia coli. J Bacteriol 188:8360-8367

De la Cruz MA, Zhao W, Farenc C, Gimenez G, Raoult D, Cambillau C, Gorvel JP, Meresse S (2013) A toxin-antitoxin module of Salmonella promote virulence in mice. PLoS Pathog 9:e1003827. doi:10.1371/journal.ppat.1003827

Engelberg-Kulka H, Glaser G (1999) Addiction modules and programmed cell death and antideath in bacterial cultures. Annu Rev Microbiol 53:43-70

Engelberg-Kulka H, Amitai S, Kolodkin-Gal I, Hazan R (2006) Bacterial programmed cell death and multicellular behavior in bacteria. PLoS Genet 2:e135. doi:10.1371/journal.pgen.0020135

Finn RD, Bateman A, Clements J, Coggill P, Eberhardt RY, Eddy SR, Heger A, Hetherington K, Holm L, Mistry J, Sonnhammer EL, Tate J, Punta M (2014) Pfam: the protein families database. Nucleic Acids Res 42:D222-D230

Garcia-Pino A, Balasubramanian S, Wyns L, Gazit E, De Greve H, Magnuson RD, Charlier D, van Nuland NA, Loris R (2010) Allostery and intrinsic disorder mediate transcription regulation by conditional cooperativity. Cell 142:101-111

Gerdes K, Maisonneuve E (2012) Bacterial persistence and toxinantitoxin loci. Annu Rev Microbiol 66:103-123

Gerdes K, Christensen SK, Lobner-Olesen A (2005) Prokaryotic toxin-antitoxin stress response loci. Nat Rev Microbiol 3:371-382

Germain E, Castro-Roa D, Zenkin N, Gerdes K (2013) Molecular mechanism of bacterial persistence by HipA. Mol Cell $52: 248-254$

Goeders N, Van Melderen L (2014) Toxin-antitoxin systems as multilevel interaction systems. Toxins (Basel) 6:304-324

Guglielmini J, Van Melderen L (2011) Bacterial toxin-antitoxin systems: translation inhibitors everywhere. Mob Genet Elements 1:283-290

Harms A, Stanger FV, Scheu PD, de Jong IG, Goepfert A, Glatter T, Gerdes K, Schirmer T, Dehio C (2015) Adenylylation of Gyrase and Topo IV by FicT toxins disrupts bacterial DNA topology. Cell Reports 12:1497-1507. doi:10.1016/j.celrep.2015.07.056

Harvey RM, Stroeher UH, Ogunniyi AD, Smith-Vaughan HC, Leach AJ, Paton JC (2011) A variable region within the genome of Streptococcus pneumoniae contributes to strain-strain variation in virulence. PLoS ONE 6:e19650. doi:10.1371/journal. pone.0019650

Jiang Y, Pogliano J, Helinski DR, Konieczny I (2002) ParE toxin encoded by the broad-host-range plasmid RK2 is an inhibitor of Escherichia coli gyrase. Mol Microbiol 44:971-979

Kaspy I, Rotem E, Weiss N, Ronin I, Balaban NQ, Glaser G (2013) HipA-mediated antibiotic persistence via phosphorylation of the glutamyl-tRNA-synthetase. Nature communications 4:3001. doi:10.1038/ncomms4001

Kim Y, Wang X, Ma Q, Zhang XS, Wood TK (2009) Toxin-antitoxin systems in Escherichia coli influence biofilm formation through YjgK (TabA) and fimbriae. J Bacteriol 191:1258-1267

Korch SB, Henderson TA, Hill TM (2003) Characterization of the hipA7 allele of Escherichia coli and evidence that high persistence is governed by (p)ppGpp synthesis. Mol Microbiol 50:1199-1213 
Leplae R, Geeraerts D, Hallez R, Guglielmini J, Dreze P, Van Melderen L (2011) Diversity of bacterial type II toxin-antitoxin systems: a comprehensive search and functional analysis of novel families. Nucleic Acids Res 39:5513-5525

Loris R, Garcia-Pino A (2014) Disorder- and dynamics-based regulatory mechanisms in toxin-antitoxin modules. Chem Rev 114:6933-6947

Magnuson RD (2007) Hypothetical functions of toxin-antitoxin systems. J Bacteriol 189:6089-6092

Makarova KS, Wolf YI, Koonin EV (2009) Comprehensive comparative-genomic analysis of type 2 toxin-antitoxin systems and related mobile stress response systems in prokaryotes. Biology direct 4:19. doi:10.1186/1745-6150-4-19

Maki S, Takiguchi S, Miki T, Horiuchi T (1992) Modulation of DNA supercoiling activity of Escherichia coli DNA gyrase by F plasmid proteins. Antagonistic actions of LetA (CcdA) and LetD (CcdB) proteins. J Biol Chem 267:12244-12251

Masuda H, Tan Q, Awano N, Wu KP, Inouye M (2012) YeeU enhances the bundling of cytoskeletal polymers of MreB and FtsZ, antagonizing the CbtA (YeeV) toxicity in Escherichia coli. Mol Microbiol 84:979-989

Mutschler H, Gebhardt M, Shoeman RL, Meinhart A (2011) A novel mechanism of programmed cell death in bacteria by toxin-antitoxin systems corrupts peptidoglycan synthesis. PLoS Biol 9:e1001033. doi:10.1371/journal.pbio.1001033

Nariya H, Inouye M (2008) MazF, an mRNA interferase, mediates programmed cell death during multicellular Myxococcus development. Cell 132:55-66

Ogura T, Hiraga S (1983) Mini-F plasmid genes that couple host cell division to plasmid proliferation. Proc Natl Acad Sci USA 80:4784-4788
Rocker A, Meinhart A (2015) A cis-acting antitoxin domain within the chromosomal toxin-antitoxin module EzeT of Escherichia coli quenches toxin activity. Mol Microbiol 97:589-604

Sberro H, Leavitt A, Kiro R, Koh E, Peleg Y, Qimron U, Sorek R (2013) Discovery of functional toxin/antitoxin systems in bacteria by shotgun cloning. Mol Cell 50:136-148

Schuster CF, Bertram R (2013) Toxin-antitoxin systems are ubiquitous and versatile modulators of prokaryotic cell fate. FEMS Microbiol Lett 340:73-85

Sevin EW, Barloy-Hubler F (2007) RASTA-Bacteria: a web-based tool for identifying toxin-antitoxin loci in prokaryotes. Genome Biol 8:R155

Shao Y, Harrison EM, Bi D, Tai C, He X, Ou HY, Rajakumar K, Deng Z (2011) TADB: a web-based resource for Type 2 toxinantitoxin loci in bacteria and archaea. Nucleic Acids Res 39:D606-D611

Tabone M, Ayora S, Alonso JC (2014) Toxin zeta reversible induces dormancy and reduces the UDP- $N$-acetylglucosamine pool as one of the protective responses to cope with stress. Toxins (Basel) 6:2787-2803

Van Melderen L (2010) Toxin-antitoxin systems: why so many, what for? Curr Opin Microbiol 13:781-785

Van Melderen L, Saavedra De Bast M (2009) Bacterial toxinantitoxin systems: more than selfish entities? PLoS Genet 5:e1000437. doi:10.1371/journal.pgen.1000437

Wang X, Wood TK (2011) Toxin-antitoxin systems influence biofilm and persister cell formation and the general stress response. Appl Environ Microbiol 77:5577-5583

Yamaguchi Y, Park JH, Inouye M (2011) Toxin-antitoxin systems in bacteria and archaea. Annu Rev Genet 45:61-79 